\title{
Processing Speed in Childhood and Adolescence: Longitudinal Models for Examining Developmental Change
}

\author{
Robert V. Kail \\ Purdue University
}

\author{
Emilio Ferrer \\ University of California at Davis
}

\begin{abstract}
The primary aim of the present study was to examine longitudinal models to determine the function that best describes developmental change in processing speed during childhood and adolescence. In one sample, children and adolescents $(N=503)$ were tested twice over an average interval of 2 years on two psychometric measures of processing speed: Visual Matching and Cross Out. In another sample, children and adolescents $(N=277)$ were tested four times, every 6 months, on Cross Out. Age-related changes in performance on both tasks were examined using six longitudinal models representing different hypotheses of growth. Linear, hyperbolic, inverse regression, and transition models yielded relatively poor fit to the data; the fit of the exponential and quadratic models was substantially better. The heuristic value of these latter models is discussed.
\end{abstract}

The speed with which children and adolescents execute basic cognitive processes (hereafter, processing speed) consistently predicts performance on a variety of cognitive tasks. More rapid processing is associated, for example, with increased capacity of working memory, enhanced inductive reasoning, and greater accuracy in solving arithmetic word problems (Fry \& Hale, 1996; Kail \& Hall, 1999; Kail, 2007).

To understand the nature of age-related change in processing speed and its links to other cognitive processes, researchers have investigated the defining properties of processing speed. One of these is the developmental profile associated with increased processing speed: Processing speed increases substantially in early and middle childhood, continues to increase though not as rapidly in late childhood and early adolescence, and reaches asymptotic values in mid-to-late adolescence (Kail, 1991). Identifying the mathematical function(s) that describes this pattern of change has been an important goal of research because such a function would yield a precise description of change and could provide insights into the mechanisms underlying developmental change.

Illustrating this type of research is a study by Kail (1986, experiment 1) in which individuals at 12 ages-8 to 15 years and 18-21 years-judged

We thank John J. McArdle for sharing the WJ-R retest data with us. This research was supported by grants from the National Institute of Child Health and Human Development (R01 - R0046927) and the National Science Foundation (SBR 93019 and BCS 27766). Portions of these data were presented at the 2004 meeting of the American Psychological Society, Chicago.

Correspondence concerning this article should be addressed to Robert V. Kail, Department of Psychological Sciences, Purdue University, West Lafayette, IN 47907 . Electronic mail may be sent to rkail@purdue.edu. whether pairs of pictures were identical physically or in name. Response times for these judgments were used to estimate the time needed to retrieve object names from long-term memory. Retrieval times declined substantially with age, with values of 303 , 147, and 99 ms for 8-, 11-, and 14-year-olds, respectively.

To characterize these changes, exponential and hyperbolic functions were chosen because they had been used successfully to describe changes due to learning and practice. Traditionally, exponential functions are associated with replacement models of learning in which incorrect responses gradually replace correct ones and hyperbolic functions are associated with accumulation models in which correct responses accumulate in the total pool, ultimately swamping incorrect answers by sheer numbers (Mazur \& Hastie, 1978; Newell \& Rosenbloom, 1981). Kail (1986) found that the exponential function provided a better fit to the data than did the hyperbolic, an outcome consistent with replacement models of learning.

Several subsequent studies (e.g., Canfield, Smith, Brezsnyak, \& Snow, 1997; Fry \& Hale, 1996; Kail, 1988, 1991; Miller \& Vernon, 1997) also found that agerelated change in processing speed is well characterized by an exponential function. Nevertheless, the evidence is far from compelling, for two reasons. First, virtually all studies of developmental change in processing speed have been cross-sectional. We know of only two studies that have reported longitudinal evidence concerning the shape of the function that

(C) 2007 by the Society for Research in Child Development, Inc. All rights reserved. 0009-3920/2007/7806-0009 
characterizes growth in processing speed and neither focused specifically on change in childhood and adolescence. In one of these studies, Canfield et al. examined processing speed during infancy and found support for nonlinear change between 2 and 12 months. McArdle, Ferrer-Caja, Hamagami, and Woodcock (2002) studied lifespan changes in cognitive functioning (including processing speed). They concluded that the change in processing speed in childhood and adolescence was nonlinear, but their models did not focus specifically on these ages. The absence of relevant longitudinal evidence on the shape of developmental functions is, of course, problematic because the best-fitting function for group data may not provide the best characterization of individuals' data.

Second, cross-sectional studies have focused almost exclusively on the descriptive use of these functions and ignored their implications for underlying developmental mechanisms. That is, as noted previously, in research on change due to practice and learning, exponential functions are associated with replacement models in which learning reflects the gradual replacement of incorrect responses with correct responses. The manner by which age would yield such replacement, or, for that matter, what such replacement would mean in the context of speeded processes, has not been considered.

Our primary aim in this study was to use longitudinal data to investigate the shape of the function that characterizes age-related change in processing speed. To operationalize processing speed, we selected two psychometric measures-Visual Matching and Cross Out - taken from the Woodcock-Johnson Psychoeducational Battery-Revised (WJ-R). These measures define a processing speed construct on the WJ-R and have been used frequently to estimate processing speed in developmental work (e.g., McBride-Chang \& Kail, 2002). We used two longitudinal samples: in one, children and adolescents were tested twice on Visual Matching and Cross Out, and in the other, children and adolescents were tested four times on Cross Out only. Although two or four points are not optimal for determining best-fitting functions for each person, in the context of a cohort-sequential design, they can be sufficient to represent individual growth functions together with information relative to individual variations in such growth (Ferrer \& McArdle, 2004; McArdle et al., 2002).

A secondary aim of our study was to use information concerning the fit of the different models to generate hypotheses regarding mechanisms underlying developmental change in processing speed. Consequently, we compared the fit provided by a range of different functions that have been useful in prior studies of behavioral and neural development and that embodied different assumptions regarding underlying mechanisms. Thus, by identifying the best-fitting models, we aimed to provide a precise description of longitudinal change in processing speed and to gain insights into plausible mechanisms giving rise to those changes.

\section{Age-Based Mixed Growth Models}

To examine changes in processing speed over time, we used a series of mixed models (also known as hierarchical linear or multilevel models) that included age as the underlying time dimension (Table 1). We chose models that had been investigated in prior cross-sectional work as well as additional models that seemed to be potentially useful to capture the nonlinear changes in processing speed observed during the target years.

Consider a variable $Y$ measured on a person $(i=1$ to $I$ ) at a measurement occasion $(t=1$ to $T)$. A basic linear growth model can be written as:

$$
Y_{i t}=\beta_{0 i}+\beta_{1 i} \cdot \text { age }_{i t}+e_{i t},
$$

where $Y_{i t}$ is the observed score on person $i$ at measurement $t, \beta_{0 i}$ represents the coefficient associated with the intercept for person $i, \beta_{1 i}$ represents the coefficient associated with the linear slope for person $i$, age $i_{i t}$ is the observed age of person $i$ at measurement $t$, and $e_{i t}$ is the error score of person $i$ at measurement $t$. As denoted by the subscript $i$, this model includes sources of individual differences in the intercept and slope, whose terms can be decomposed at a second level as:

$$
\beta_{0 i}=v_{0}+u_{0 i} \text { and } \beta_{1 i}=v_{1}+u_{1 i}
$$

indicating that the intercept and the slope scores have fixed group means $\left(v_{0}\right.$ and $\left.v_{1}\right)$ and residuals $\left(u_{0 i}\right.$ and $\left.u_{1 i}\right)$, and these residuals have zero means and variance components $\left(\sigma_{0}^{2}, \sigma_{0}^{2}\right.$, and $\left.\sigma_{01}\right)$. Similarly, the error term associated with the within-person residual $e_{i t}$ has also a zero mean and a variance term $\sigma_{e}^{2}$.

More generally, the vectors corresponding to the between-individual residuals $\mathbf{u}_{i}$ and the individual error residuals $\mathbf{e}_{i}$ are assumed to be independent and normally distributed, that is, $\mathbf{u}_{i} \sim N(0, \mathbf{G})$ and $\mathbf{e}_{\boldsymbol{i}} \sim$ $N(0, \mathbf{R})$. To test specific hypotheses or due to data constraints, restrictions can be imposed as needed in any of these covariance matrices. In this model, we did not impose any restriction and estimated all six parameters (i.e., two fixed effects, three random effects, and one residual term). 
Table 1

Algebraic Expressions of Models Fitted to the Visual Matching and Cross Out Scores

\begin{tabular}{|c|c|c|}
\hline Model (no. parameters) & Equation & Parameters \\
\hline Linear (6) & $\begin{array}{l}Y_{i t}=\beta_{0 i}+\beta_{1 i} \cdot \text { age }_{i t}+e_{i t} \\
\beta_{0 i}=v_{0}+u_{0 i}, \beta_{1 i}=v_{1}+u_{1 i}\end{array}$ & $\beta_{0}=$ intercept,$\beta_{1}=$ linear slope \\
\hline Quadratic (8) & $\begin{array}{l}Y_{i t}=\beta_{0 i}+\beta_{1 i} \cdot \text { age }_{i t}+\beta_{2 i} \cdot \text { age }_{i t}^{2}+e_{i t} \\
\beta_{0 i}=v_{0}+u_{0 i}, \beta_{1 i}=v_{1}+u_{1 i}, \beta_{2 i}=v_{2}+u_{2 i}\end{array}$ & $\begin{array}{l}\beta_{0}=\text { intercept, } \beta_{1}=\text { linear } \\
\text { slope, } \beta_{2}=\text { quadratic slope }\end{array}$ \\
\hline Exponential (7) & $\begin{array}{l}Y_{i t}=\beta_{0 i}+\beta_{1 i} \cdot e^{-c^{*} \text { age }}+e_{i t} \\
\beta_{0 i}=v_{0}+u_{0 i}, \beta_{1 i}=v_{1}+u_{1 i}\end{array}$ & $\begin{array}{c}\beta_{0}=\text { asymptote, } \beta_{1}=\text { intercept } \\
\quad\left(\text { with } \beta_{0}\right), c=\text { rate of growth }\end{array}$ \\
\hline Hyperbolic (7) & $\begin{array}{l}Y_{i t}=\beta_{0 i}-\left(\beta_{1 i} /\left(\text { age }_{i t}+c\right)\right)+e_{i t} \\
\beta_{0 i}=v_{0}+u_{0 i}, \beta_{1 i}=v_{1}+u_{1 i}\end{array}$ & $\begin{array}{l}\beta_{0}=\text { asymptote } \\
\quad\left(\beta_{1} /(\text { age }+c)\right)=\text { rate } \\
\text { of approach to asymptote }\end{array}$ \\
\hline Inverse regression (6) & $\begin{array}{l}Y_{i t}=\beta_{0 i}+\left(\beta_{1 i} / \text { age }_{i t}\right)+e_{i t} \\
\beta_{0 i}=v_{0}+u_{0 i}, \beta_{1 i}=v_{1}+u_{1 i}\end{array}$ & $\begin{array}{l}\beta_{0}=\text { asymptote } \\
\quad\left(\beta_{1} / \text { age }\right)=\text { rate of approach } \\
\text { to asymptote }\end{array}$ \\
\hline Latent transition (8) & $\begin{array}{l}Y_{i t}=\beta_{0 i}+\beta_{1 i} \text { age }_{i t}+\delta_{1 i}\left(\operatorname{age}_{i t}-\tau\right)+e_{i t} \\
\beta_{0 i}=v_{0}+u_{0 i}, \beta_{1 i}=v_{1}+u_{1 i}, \delta_{1 i}=v_{2}+u_{2 i}\end{array}$ & $\begin{array}{l}\beta_{0}=\text { intercept, } \beta_{1}=\text { linear } \\
\quad \text { slope before transition, } \beta_{1}-\delta_{1}=\text { linear } \\
\text { slope after transition, } \tau=\text { transition point }\end{array}$ \\
\hline All models & $\mathbf{u}_{i} \sim N(0, \mathbf{G}) ; \mathbf{e}_{\boldsymbol{i}} \sim N(0, \mathbf{R})$ & $\begin{array}{l}\mathbf{G}=\text { covariance } \\
\text { matrix of random } \\
\text { effects, } \mathbf{R}=\text { matrix } \\
\text { of residual variances }\end{array}$ \\
\hline
\end{tabular}

Note. See text for explanation of all model parameters.

The second selected model was based on the exponential function studied by Kail $(1986,1988)$, expressed here as:

$$
Y_{i t}=\beta_{0 i}+\beta_{1 i} \cdot e^{-c^{*} \text { age }}+e_{i t},
$$

where $\beta_{0 i}$ now represents the asymptote for person $i$, $\beta_{1 i}$ represents the intercept (together with $\beta_{0}$ ), $e$ is the base of natural logarithms, and $c$ represents a rate of growth. Here, we modeled individual differences in the coefficients $\beta_{0 i}$ and $\beta_{1 i}$, yielding a $2 \times 2$ covariance matrix of random effects $G$ with three parameters. We did not impose any restriction in this model and estimated all seven parameters (i.e., three fixed effects, three random effects, and one residual term).

The third model was the hyperbolic function evaluated by Kail $(1986,1988)$, expressed here as:

$$
Y_{i t}=\beta_{0 i}-\left(\beta_{1 i} /\left(\operatorname{age}_{i t}+c\right)\right)+e_{i t},
$$

where, as before, $\beta_{0 i}$ represents the asymptote for person $i$ and the term $\left(\beta_{1 i} /(\right.$ age $\left.+c)\right)$ represents the rate with which person $i$ reaches asymptote. As in the exponential model, here we considered individual differences in the coefficients $\beta_{0 i}$ and $\beta_{1 i}$, also yielding a $2 \times 2$ covariance matrix of random effects $G$ with three parameters. We estimated all seven parameters of the model (i.e., three fixed effects, three random effects, and one residual term).

The fourth model was an inverse regression function investigated by Luna, Garver, Urban, Lazar, and Sweeney (2004) in a study of the speed with which 8to 30-year-olds move their eyes to fixate a target in the peripheral visual field. In this cross-sectional study, age-related changes in saccadic latencies were best described by an inverse regression function. In our analyses, we expressed this model as:

$$
Y_{i t}=\beta_{0 i}+\left(\beta_{1 i} / \text { age }_{i t}\right)+e_{i t},
$$

where $\beta_{0 i}$ represents the asymptote for person $i$ and the term $\left(\beta_{1 i} /\right.$ age $)$ represents the rate with which person $i$ reaches asymptote. We also considered individual differences in the coefficients $\beta_{0 i}$ and $\beta_{1 i}$, yielding a $2 \times 2$ covariance matrix of random effects with three parameters. Because we did not impose any restriction, this model yielded six parameters (i.e., two fixed effects, three random effects, and one residual term).

The fifth model, a quadratic function, has been used to characterize physical and neural growth during childhood and adolescence. For example, increases in total body fat between 8 and 20 years of age are well described by a quadratic function 
(Guo, Chumlea, Roche, \& Siervogel, 1997). In the same vein but more directly related to the present work, volume of cortical gray matter, as measured from magnetic resonance imaging, increases in childhood but decreases in adolescence, in a manner that is well described by a quadratic function (Giedd et al., 1999). Because physical growth generally and neural growth in particular may enhance processing speed, we chose the quadratic function to fit our longitudinal data. We wrote the quadratic model as:

$$
Y_{i t}=\beta_{0 i}+\beta_{1 i} \cdot \text { age }_{i t}+\beta_{2 i} \cdot \text { age }_{i t}^{2}+e_{i t},
$$

where age $e_{i t}^{2}$ is age squared, $\beta_{2 i}$ is the regression coefficient associated with the quadratic slope score of person $i$ at time $t$, and the other terms are as defined in Equation 1. As described in Table 1, this model includes sources of individual differences in the intercept, linear slope, and quadratic slope. Thus, the covariance matrix of random effects $\mathrm{G}$ of this model is a $3 \times 3$ matrix that includes six parameters. To avoid comparisons between this and other models that would rely on the differences in random components, we imposed restrictions in the $\mathbf{G}$ matrix of the quadratic model. In particular, we fixed to zero the covariances between the term of the quadratic slope and the terms of the intercept and the linear slope. The remaining eight parameters (i.e., three fixed effects, four random effects, and one residual term) were freely estimated.

Finally, we also examined a transition model derived from the work by Cudeck and Klebe (2002). They showed that many patterns of nonlinear change are characterized by discontinuities in which the change function takes on different values before and after a transition point. For example, the developmental function might be linear before and after transition but with a much steeper slope before transition; aggregating across individuals would produce a nonlinear function. This model can be seen as a variant of a spline model in which the transition point is unknown. Spline models have been used in developmental work to examine nonlinear changes typically observed in these years (e.g., Ferrer \& McArdle, 2004; McArdle et al., 2002; Ferrer et al., in press). In the present case, we investigated a model in which processing speed changes linearly before and after a transition but much more rapidly before.

The transition model (Cudeck \& Klebe, 2002) can be written as:

$$
Y_{i t}=\beta_{0 i}+\beta_{1 i} \cdot \operatorname{age}_{i t}+\delta_{1 i}\left(\operatorname{age}_{i t}-\tau\right)+e_{i t},
$$

where $\beta_{0 i}$ represents the coefficient associated with the intercept, $\beta_{1 i}$ represents the coefficient associated with the linear slope before the transition, $\delta_{1 i}$ represents the difference between the slopes before and after the transition (thus, $\beta_{1}-\delta_{1}$ equals the second slope), and $\tau$ represents the latent transition point. As was the case for the quadratic model, we modeled individual differences in three parameters, yielding a $3 \times 3 \mathrm{G}$ matrix with six parameters. Here, we set to zero all covariances in the matrix and estimated the remaining three variance components. In total, we estimated eight parameters in this model (i.e., four fixed effects, three random effects, and one residual term).

The models depicted in Equations 1-7 were fitted to the data from the two-occasion samples. However, the four-occasion sample provided sufficient data for us to evaluate effects associated with retest (i.e., changes related to repeated measurements), in addition to the age-related effects. For this purpose, Equations 1-7 were modified by adding components representing the retest effects. For example, in the case of a linear model, the new specification resulted in the following equation:

$$
Y_{i t}=\beta_{0 i}+\beta_{1 i} \cdot \text { age }_{i t}+\beta_{2 i} \cdot \text { retest }_{i t}+e_{i t},
$$

where retest $t_{i t}$ represents the effects related to measurement occasion of person $i$ at assessment $t$. The other terms are as specified in Equation 1. In this model, change in $Y$ is described as a function of two processes that unfold over time: age and retest. The first process age $e_{i t}$ varies over time for each person, so this term represents an age-based growth process. It is a slope based on age at testing occasion $t$ (i.e., average change in $Y$ per year for a person $i$ ). The retest component, retest ${ }_{i t}$, in turn represents a growth process based on the measurement occasion (i.e., average change in $Y$ per unit change in retest for a person $i$; see Ferrer, Salthouse, Stewart, \& Schwartz, 2004).

To summarize, in the present study, we obtained psychometric measures of processing speed for two samples of children and adolescents, each of whom was tested either two or four times. The resulting longitudinal data were used to evaluate the fit of six potential developmental functions, thereby providing evidence regarding the best description of the change as well as the underlying processes.

\section{Method}

\section{Samples}

The two-occasion sample included 272 boys and 231 girls, each tested twice on Visual Matching and Cross Out. The mean ages at first and second testing were 9.56 and 11.86 years, respectively $(S D=3.1$ and 2.85 years, respectively). Of these, 344 (159 females) 
were drawn from the norming sample for the WJ-R tests and were then retested (McArdle et al., 2002). We included all individuals who were at least 5 years old when tested initially and no older than 18 years when retested. The remaining 159 children and adolescents had participated originally in studies by the first author. Specifically, of the 128 U.S. children tested by McBride-Chang and Kail (2002), 46 (21 females) attending one elementary school were retested approximately 3.5 years later and 27 (11 females) attending a second elementary school were tested approximately 6 years later. In addition, from children who participated in studies by Kail, Hall, and Caskey (1999) and Kail (1997), we recruited 86 children (40 females) who were 8-11 years old when first tested; they were retested $12-15$ months later.

The four-occasion sample included 277 children (140 females). Of these, 240 were tested on all four occasions, 5 tested thrice, 7 tested twice, and 25 tested once. They were tested every 6 months, with mean ages at first, second, third, and fourth testing of 9.25, $9.77,10.29$, and 10.82 years, respectively $(S D=1.99$, $2.00,2.01$, and 1.99 years, respectively).

\section{Measures}

The Visual Matching and Cross Out tests from the WJ-R were used to estimate processing speed. In the former, each of 60 rows includes six digits, two of which are identical (e.g., 89529 7); the participant circles the identical digits. The performance measure is the number of rows completed correctly in $3 \mathrm{~min}$. In the latter, each of 30 rows consists of a geometric figure at the left end of a row and 19 similar figures to the right. One row, for example, consists of a triangle enclosing a single dot; the 19 figures are triangles with various objects in the interior (e.g., a single dot, three dots, a plus, a square). The child places a line through the 5 figures of the 19 that are identical to the one at the left. The performance measure is the number of rows completed in $3 \mathrm{~min}$.

\section{Procedure}

All participants were tested individually. Individuals in the norming sample completed Visual Matching and Cross Out along with other subtests from the WJ-R. Individuals in the other samples typically completed Visual Matching and Cross Out along with other measures of working memory and reasoning. For individuals in the four-occasion sample, the actual version of Cross Out was administered on the first and fourth occasions; on the second and third occasions, modified versions were used in which items had been randomly reordered within each row and rows had been randomly reordered on a page. (Different modified versions were used on the second and third occasions.) They were also tested on measures of memory and reasoning, but Cross Out was always administered first.

\section{Results}

For both measures, the familiar developmental pattern was evident: Performance improved across childhood and adolescence but more rapidly in childhood. This pattern is manifest in Figure 1, which depicts aggregated data obtained by dividing each sample into 10 subsamples by age (i.e., the first subsample consisted of the children in the bottom age decile at the first testing). Nonlinear change is evident for Visual Matching and Cross Out, with both two- and four-occasion samples. Also apparent in the four-occasion data is that performance improved across sessions, though not linearly: For most agegroups, there was little change between the first and the second occasions but steady improvement in the third and fourth sessions.

The six models listed in Table 1 were fitted to the data in Figure 1 using the MIXED and NLMIXED procedures from SAS. (The SAS code for all models is available from the authors.) The fit of the models was assessed with three indexes: Akaike's information criterion (AIC), $\mathrm{AIC}_{\mathrm{c}}$, a version of $\mathrm{AIC}$ that is more appropriate with small samples, and the Bayesian information criterion. All measures are derived from the log-likelihood ratio that characterizes the fit of the model to the data; each adjusts the degree of fit based on the number of parameters in the model, and in all cases, smaller values indicate a better fit. The pattern of results was the same with all indexes; consequently, we present only the findings for AIC.

The fit of the six models, expressed in terms of AIC values, is shown in Table 2, separately for each test, and for Cross Out, separately for the two- and fouroccasion samples. The quadratic model provided the best fit in two instances and the transition model in one. The linear, hyperbolic, and inverse regression models consistently provided the worst fit. Because the models are not nested, they cannot be compared with likelihood ratio tests; instead, we used information theoretic methods to compare their relative fit. We first computed the difference in fit, $\triangle \mathrm{AIC}$, by subtracting the value of AIC for the best-fitting model from the AIC value for each of the other models. By definition, $\triangle \mathrm{AIC}=0$ for the best-fitting model and $\Delta$ AIC $>0$ for all others. Next, we computed an 

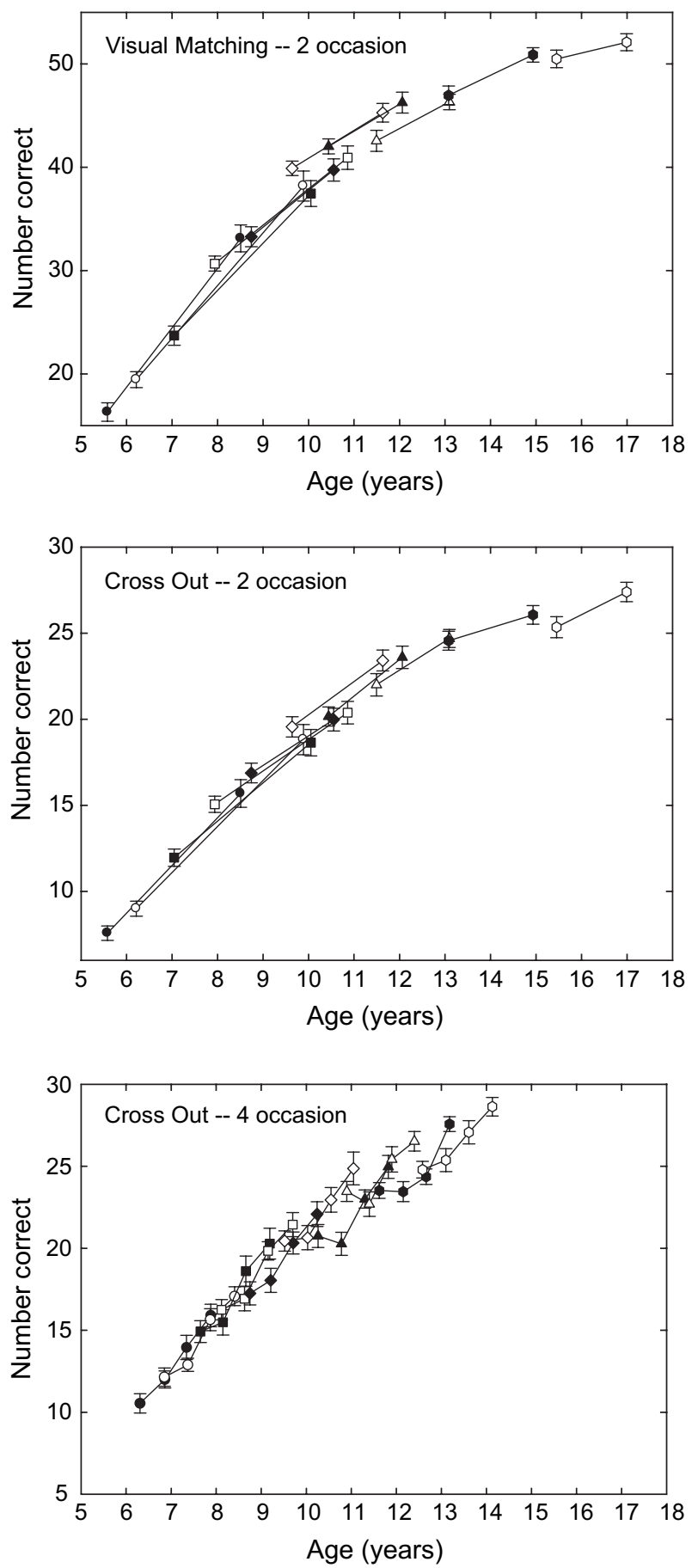

Figure 1. Age-related change in performance on Visual Matching and Cross Out tasks, with performance on the latter task presented separately for two- and four-occasion samples. In each panel, the data are presented for 10 groups of participants based on age when tested initially. For example, the first group (i.e., filled circles) consists of the youngest $(10 \%)$ participants and the last group (i.e., the open hexagons), the oldest $(10 \%)$. For the two-occasion sample, each group includes 50-51 participants and for the fouroccasion sample, 27-28 participants. Error bars depict standard errors.
Akaike weight, $w$, for each model, which is defined as:

$$
w=e^{-1 / 2 \Delta \mathrm{AIC}} / \Sigma e^{-1 / 2 \Delta \mathrm{AIC}}
$$

and indicates the relative likelihood of the model given the data and other models under consideration (Burnham \& Anderson, 2002; Wagenmakers \& Farrell, 2004).

The $w$ values, shown in Table 2, reveal that the linear, inverse regression, and hyperbolic models do not fit the data well. Across both measures and samples, $w \leq .056$ for these models, indicating that they are highly unlikely descriptions of these data. Findings for the other three models are less consistent. For the Visual Matching and two-occasion Cross Out data, the quadratic provides the best fit, with $w \geq .70$. However, for the four-occasion Cross Out data, the transition model provides the best fit, with $w=.616$. In other words, the outcomes of model fitting were consistent across measures for the two-occasion sample, but the results for Cross Out varied across the two- and four-occasion samples.

One plausible explanation of these differences is the fact that the two-occasion sample included a broader range of ages (5-17 years at Time 1 compared to 6-13.5 years at Time 1 in the fouroccasion sample). Perhaps, the quadratic provides a better fit when the sample includes a larger segment of adolescence, a time when processing speed is reaching asymptote. To evaluate this possibility, we randomly selected 277 cases from the two-occasion sample, combined them with the 277 cases from the four-occasion sample, and fitted the models to these pooled data. The results, shown in the last set of columns in Table 2, yielded outcomes that are consistent with those obtained for the two-occasion sample alone. That is, the quadratic model provided the best fit and the exponential model provided the second best fit. Thus, it seems that across the entire range of childhood and adolescence, the quadratic model provides the best characterization of age-related change in processing speed; the fit of this model to the data is shown in Figure 2.

To evaluate the more plausible models further, we determined the extent to which either of them systematically mispredicted participants' scores. That is, a close fit to the data might be achieved, for example, by consistently overestimating actual scores at one age but consistently underestimating them at another. To evaluate this possibility, we calculated residuals for each child's scores (i.e., actual score - predicted score), separately for the quadratic, exponential, and transitional models. To determine whether these residuals 


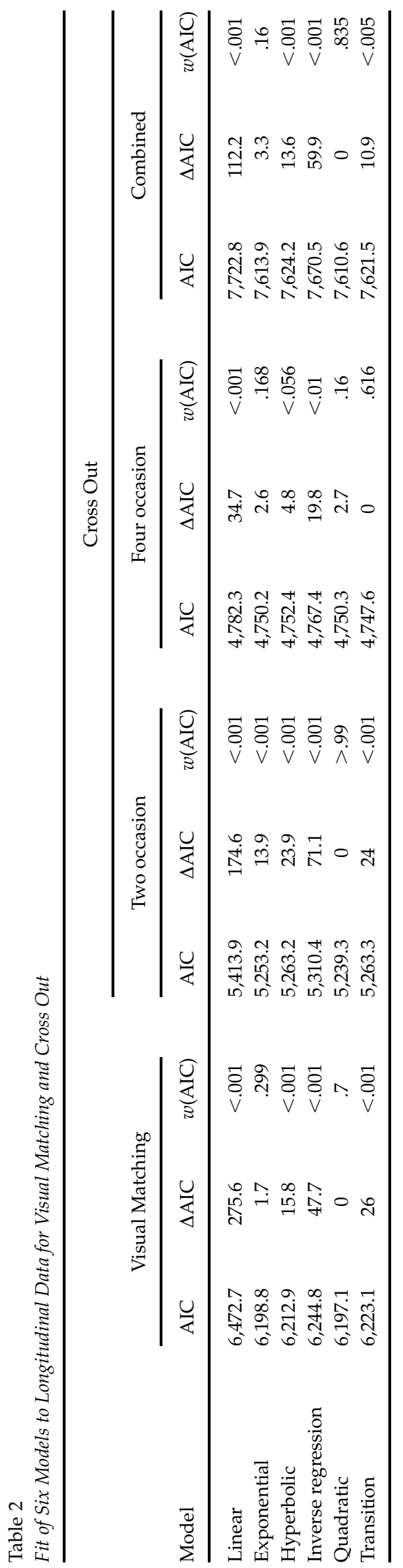

deviated systematically from 0 , we correlated them with age, separately for each testing occasion.

The results are shown in Table 3. For Visual Matching, none of the correlations is significant. In contrast, for Cross Out, three of the six correlations are significant for the quadratic and two of six are significant for the exponential. In these cases, the quadratic and exponential functions tend to overestimate scores at younger ages at Time 1 but overestimate them at Time 2 for the two-occasion sample and at Time 4 for the four-occasion sample. However, these correlations are very small, suggesting that these predictive errors are minor.

Another way to compare these three models is in the consistency of parameter estimates across samples. A model might achieve a particularly good fit by modeling a local feature of the data particularly well (i.e., a feature specific to a particular sample and not characteristic of a larger population). If this were true, then estimated values of parameters should vary across samples, reflecting adjustments to samplespecific local features. If, instead, the fit of a model is driven by basic features of the underlying population, then estimated values of a model's parameters should be consistent across samples drawn from that population.

We compared the consistency of parameter estimates for the quadratic and exponential models for Cross Out scores across the two- and four-occasion samples. These estimates are presented in Table 4 . In fact, the parameter estimates for the quadratic and the exponential functions are consistent across the twoand four-occasion samples. That is, in no case do estimated values of parameters for these models differ across the two samples.

\section{Discussion}

Our primary goal in this study was to use developmental data and longitudinal models to identify the function that best characterizes age-related change in processing speed. Our secondary goal was to use the findings from curve fitting to generate hypotheses regarding mechanisms responsible for growth of processing speed. In this section, we discuss each of these goals in turn.

\section{Functions That Best Characterize Development of Processing Speed}

We analyzed a set of six plausible developmental models; the results indicate that three are not reasonable representations of these developmental data. The 

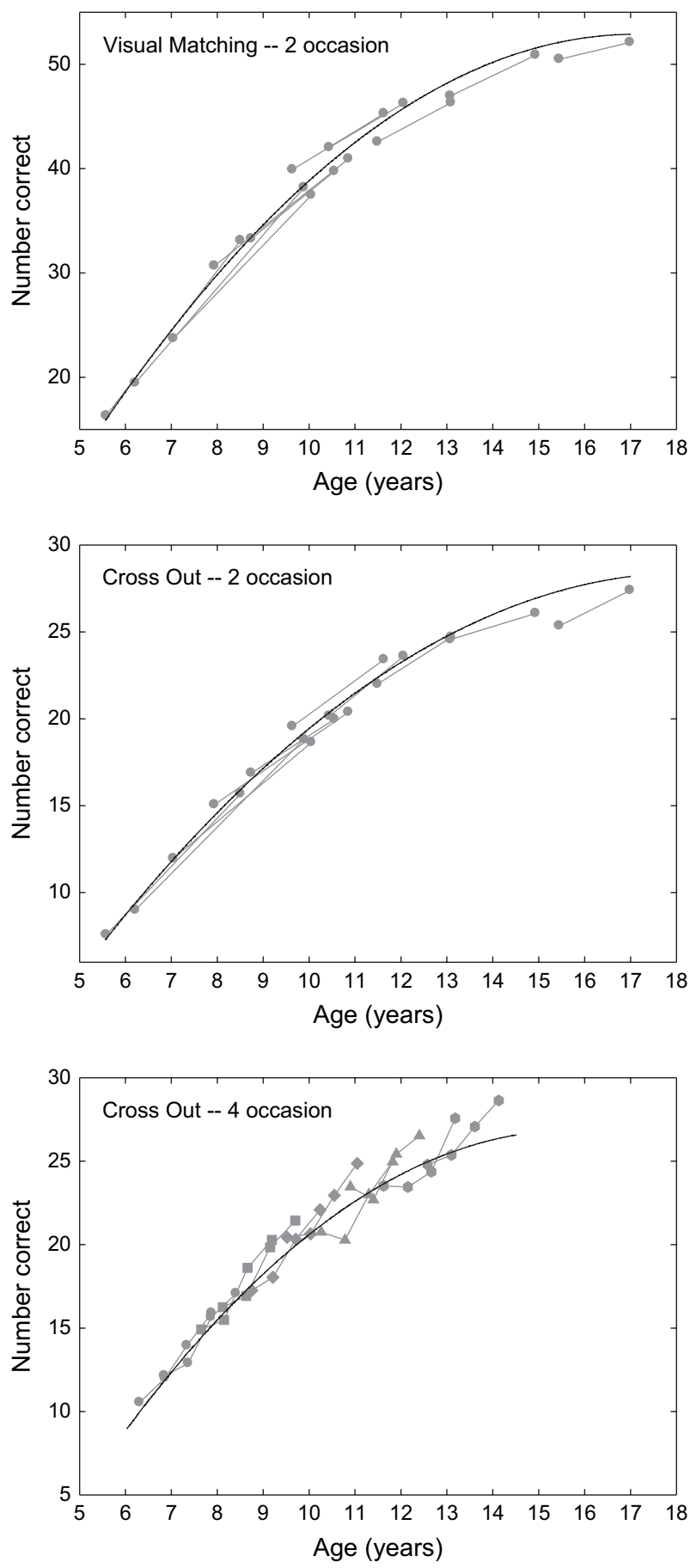

Figure 2. The fit of the quadratic model to the Visual Matching and Cross Out scores (from Figure 1).

linear model was included primarily as a reference point, although aggregating across individual linear functions in cross-sectional work could yield an aggregate nonlinear function. However, the linear function consistently provided the poorest fit to the data. Evidently, processing speed does not increase linearly across childhood and adolescence.
Table 3

Correlations Between Age and Residuals

\begin{tabular}{lccc}
\hline & & \multicolumn{2}{c}{ Model } \\
\cline { 3 - 4 } Measure & Time & Quadratic & Exponential \\
\hline Cross Out & 1 & $-.104^{*}$ & $-.090^{*}$ \\
& 2 & $.106^{*}$ & .073 \\
Cross Out & 1 & .045 & .020 \\
& 2 & -.060 & -.056 \\
& 3 & -.105 & -.089 \\
Visual Matching & 4 & $.142^{*}$ & $.158^{*}$ \\
& 1 & -.042 & -.019 \\
& 2 & .044 & .020 \\
\hline
\end{tabular}

$* p<.05$

The other models that received virtually no support were the hyperbolic and the inverse regression. Across the three sets of data, the odds that these models provided the best fit never exceeded 0.022 . The lack of evidence for the hyperbolic model confirms the cross-sectional results reported by Kail (1986, 1988) in which the hyperbolic consistently provided a poorer fit than did the exponential. The lack of support for the inverse regression model is surprising in light of the finding of Luna et al. (2004) that this model characterized growth in speed of saccadic eye movements. Perhaps, eye movement speed develops at a different rate than speed of cognitive processes; alternatively, this result may be an artifact of determining growth functions from cross-sectional data.

The transition model was the best-fitting model for Cross Out scores for the four-occasion sample but not when these scores were combined with Cross Out scores for the two-occasion sample. Apparently, some changes between childhood and early adolescence are well characterized by this model, but when the full range of childhood and adolescence was considered, the transition model was less accurate.

Overall, the quadratic provided the best fit to the data and the exponential provided the second best fit. The quadratic had the largest evidence weights for two of the original three data sets $(w>.7)$ and for the combined sample. Furthermore, parameters derived for this model for Cross Out were consistent across the two- and four-occasion samples. Although the exponential provided the second best fit, with parameter estimates that were consistent across two- and fouroccasion samples, the evidence weights were substantially smaller. Thus, in aggregate, the present findings point to the quadratic model as providing the best description of age-related change in processing speed between 5 and 18 years of age. 
Table 4

Parameter Estimates From Models Fitted to Cross Out Data

\begin{tabular}{|c|c|c|c|}
\hline \multirow[b]{2}{*}{ Model } & \multirow[b]{2}{*}{ Parameter } & \multicolumn{2}{|c|}{ Sample } \\
\hline & & $\begin{array}{c}\text { Two-occasion } \\
\text { data }\end{array}$ & $\begin{array}{c}\text { Four-occasion } \\
\text { data }\end{array}$ \\
\hline \multicolumn{4}{|l|}{ Quadratic } \\
\hline \multirow{3}{*}{$\begin{array}{l}\text { Fixed } \\
\text { effects }\end{array}$} & $\beta_{0}=$ intercept & $5.39(0.29)$ & $6.36(0.62)$ \\
\hline & $\beta_{1}=$ linear slope & $3.46(0.11)$ & $3.51(0.24)$ \\
\hline & $\beta_{2}=$ quadratic slope & $-0.13(0.01)$ & $-0.13(0.03)$ \\
\hline \multirow{5}{*}{$\begin{array}{r}\text { Random } \\
\text { effects }\end{array}$} & $\sigma_{0}^{2}=$ intercept & $4.40(1.30)$ & $5.98(3.15)$ \\
\hline & $\sigma_{1}^{2}=$ linear slope & $\approx 0$ & $0.01(0.01)$ \\
\hline & $\sigma_{2}^{2}=$ quadratic slope & $\approx 0$ & $=0$ \\
\hline & $\begin{array}{c}\sigma_{0,1}=\text { intercept }- \\
\text { linear slope }\end{array}$ & $0.44(0.12)$ & $0.17(0.59)$ \\
\hline & $\begin{array}{c}\sigma_{e}^{2}=\text { residual } \\
\text { variance }\end{array}$ & $4.81(0.30)$ & $3.42(0.19)$ \\
\hline \multicolumn{4}{|l|}{ Exponential } \\
\hline \multirow{3}{*}{$\begin{array}{l}\text { Fixed } \\
\text { effects }\end{array}$} & $\beta_{0}=$ asymptote & $33.76(3.07)$ & $37.69(2.79)$ \\
\hline & $\begin{array}{l}\beta_{1}=\text { intercept } \\
\quad\left(\text { with } \beta_{0}\right)\end{array}$ & $-58.25(3.74)$ & $-59.31(2.68)$ \\
\hline & $c=$ rate & $0.1403(0.0293)$ & $0.12(0.02)$ \\
\hline \multirow{4}{*}{$\begin{array}{r}\text { Random } \\
\text { effects }\end{array}$} & $\sigma_{0}^{2}=$ asymptote & $11.31(3.39)$ & $9.94(0.01)$ \\
\hline & $\sigma_{1}^{2}=$ intercept & $2.70(85.58)$ & $1.61(0.01)$ \\
\hline & $\begin{array}{l}\sigma_{0,1}=\text { asymptote- } \\
\text { intercept }\end{array}$ & $-3.37(17.19)$ & $\approx 0$ \\
\hline & $\begin{array}{c}\sigma_{e}^{2}=\text { residual } \\
\text { variance }\end{array}$ & $4.73(0.53)$ & $3.42(0.01)$ \\
\hline
\end{tabular}

Note. Standard errors are given in parentheses. The entry $\approx 0$ indicates a parameter not reliably estimated. The models for the fouroccasion data also included parameters representing retest effects. These are not included here because they were not part of the models for the two-occasion data and, thus, do not contribute to the comparison across samples.

These conclusions seem to generalize across the two psychometric measures used here. The two-occasion sample included both Visual Matching and Cross Out tasks, and the results were comparable: both tasks were best described by a quadratic model. This similar outcome is not surprising inasmuch as Visual Matching and Cross Out define the processing speed construct on the Woodcock - Johnson and correlations between them are usually substantial (e.g., for the two-occasion sample, correlations with age partialled out were .70 and .74 for Times 1 and 2, respectively). Thus, a quadratic pattern of growth seems robust across this class of psychometric tasks; we would expect similar developmental profiles for growth in processing speed as measured on similar tasks such as Number Comparisons and Identical Pictures (French, Ekstrom, \& Price, 1963).

At the same time, it is important to note that although Visual Matching and Cross Out (and similar tasks) typically define a processing speed construct on psychometric measures, this does not mean that they are "pure measures" of speed. Instead, they are complex tasks that invoke a set of perceptual, cognitive, mnemonic, and motor processes, and the developmental course for each constituent process need not necessarily be well described by the quadratic model. Nevertheless, as researchers formulate theories of the manner in which these processes are assembled to generate performance on psychometric measures, the ability to generate these best-fitting developmental functions will be one means of evaluating those theories.

\section{Hypotheses Concerning Mechanisms Underlying Performance}

Thus far, we have focused solely on the descriptive value of each model (i.e., in capturing age-related change in individuals' growth of processing speed). In this section, we focus on the heuristic value of the two best-fitting models: the quadratic and the exponential. Although the exponential was consistently a distant second in terms of fit, we include it to contrast differing ways in which models can have heuristic value.

Exponential model. The signature of exponential change-whether growth or decay-is that change occurs at a constant multiplicative rate, which means that rate is proportional to the current value of a variable. As we noted previously, exponential models have long been used to describe changes associated with learning and practice, in which performance improves rapidly at first and then continues to improve at a slower rate. Exponential change in learning and practice has typically been associated with replacement models of learning and practice in which incorrect responses are replaced by correct responses at a constant multiplicative rate over trials or time (Mazur \& Hastie, 1978).

The usual replacement model of learning does not translate readily to the speeded tasks used here because performance is essentially error-free from the outset. However, replacement need not be restricted to correct and incorrect responses. Instead, consider a system comprising elements that exist in two different states-efficient and inefficient. If the system consists entirely of inefficient elements at the outset, but elements became efficient at a constant multiplicative rate over time, the result is exponential growth to asymptote in efficient elements.

In the context of neural development, for example, the elements might be neurons, with efficient and inefficient states corresponding to myelinated and unmyelinated neurons, respectively. If unmyelinated neurons were replaced by myelinated neurons, at a constant multiplicative rate, the result would be exponential change in myelinated neurons, which might give rise to increased processing speed. 
Another possibility for this model comes from research on children's solutions to simple arithmetic problems (Shrager \& Siegler, 1998). Children tend to answer problems such as " $6+5=$ ?" in one of two ways-by retrieving an answer from long-term memory or by computing an answer using any of several strategies (e.g., counting on their fingers). In this case, retrieving an answer is efficient (fast, automatic, error-free) but computing an answer is not (slow, resource demanding, error-prone). If computed answers were replaced by retrieved answers, at a constant multiplicative rate, this would yield exponential growth in retrieved answers, which might support more rapid processing.

In fact, there is evidence suggesting that neither of these possibilities is likely to be correct, at least in the lean form described here. Based on imaging studies that determine volume of cortical white matter, change in myelination appears to be linear in childhood and adolescence, not exponential (e.g., Giedd et al., 1999). And the shift from computed answers to retrieved answers is typically considered to be a byproduct of task- and stimulus-specific experience (e.g., Rickard, 2004). It is not obvious how such an account would apply on tasks like those used here, which are novel and thus unlikely to be solved with retrieval, yet yield exponential change in performance. Nevertheless, the more general point is to show the heuristic value of exploring a class of models (i.e., replacement) that give rise to a specific pattern of change.

Quadratic model. Quadratic models represent the sum of a power function, $a x^{2}$, and a linear function, $b x$ $+c$; they are represented graphically by a parabola. In some scientific disciplines, the different parameters of the quadratic map onto distinct processes. Perhaps the most familiar, from physics, is that the height of a projectile affected only by its initial velocity and gravity is given by:

$$
Y=a t^{2}+b t+c
$$

where $t$ is time in seconds, $a=-4.9$ (rate of fall in meters), $b$ is initial upward velocity, and $c$ is initial height.

The quadratic is seldom used in this way in developmental or cognitive psychology. Indeed, we know of no models in which the parameters of the quadratic are mapped onto distinct processes. Instead, the most frequent use of the quadratic is descriptive, particularly in the case of U-shaped or inverted U-shaped relations (e.g., the inverted U-shaped function that relates recall accuracy to stress; Parker, Bahrick, Fivush, \& Johnson, 2006).

Given this state, the heuristic use of the quadratic comes in looking for other relevant domains in which growth is characterized as quadratic. As we described briefly in the introduction, physical growth in childhood and adolescence is often well described by quadratic models. More importantly, the parameters of these quadratics are often qualitatively like those obtained here: nonlinear change is achieved from a linear increase coupled with a nonlinear (power function) decrease. For example, according to the best-fitting model for the four-occasion sample, performance on Cross Out increased 3.51 rows per year but declined by -0.13 rows per year squared. Similarly, Giedd et al. (1999) reported that total cerebral volume increased $5.6 \mathrm{~cm}^{3}$ annually but declined by 0.72 cubic $\mathrm{cm}$ per year squared. Corresponding values for total body fat for girls are 1.84 and $-0.03 \mathrm{~kg}$ (Guo et al., 1997). Thus, processing speed, total cerebral volume, and total body fat all show the same pattern of quadratic change in childhood and adolescence, which suggests that all might have a common (unspecified) biological base.

A more detailed comparison of the findings of Giedd et al. (1999) provides additional clues. In fact, when cortical regions were analyzed separately, only one region had the profile of linear increase in gray matter volume coupled with a nonlinear (power function) decrease: the temporal region. (Occipital gray matter increased linearly throughout childhood and adolescence; change in front and parietal gray matter was characterized by linear and nonlinear declines.) Thus, to the extent that these declines in gray matter reflect synaptic pruning, they suggest that adult-like processing speed in mid-to-late adolescence is a byproduct of the elimination of unnecessary neural pathways, particularly in temporal cortex.

More generally, we have illustrated a second way in which curve fitting has heuristic value. When different domains, tasks, or measures share a common developmental profile-in this case, a quadratic that included linear increase and nonlinear decreasethose shared (or similar or related) elements of those domains can be used to generate hypotheses about the processes that drive development.

\section{Conclusions}

Generally, we believe that identifying a full-fledged model of the growth of processing speed requires two complementary lines of research. First, more extensive longitudinal data would provide stronger tests of the fit of the different models. The two- and fouroccasion data used here provided a preliminary assessment, but a more powerful evaluation would be possible with repeated longitudinal measurements of speed over a longer developmental span. Second, it 
would be helpful to evaluate hypotheses that emanate from the different conceptual models. For example, can the pattern of quadratic change shown here be linked directly to changes in cortical gray matter? We believe that richer developmental data and more systematic exploration of the conceptual properties of models should yield a fuller understanding of the processes that result in faster cognitive processing in childhood and adolescence, as well as the means by which increased processing speed is related to other cognitive processes.

\section{References}

Burnham, K. P., \& Anderson, D. R. (2002). Model selection and multimodel inference: A practical information-theoretic approach (2nd ed.). New York: Springer-Verlag.

Canfield, R. L., Smith, E. G., Brezsnyak, M. P., \& Snow, K. L. (1997). Information processing through the first year of life: A longitudinal study using the visual expectation paradigm. Monographs of the Society for Research in Child Development, 62,2(Serial No. 250).

Cudeck, R., \& Klebe, K. J. (2002). Multiphase mixed-effects models for repeated measures data. Psychological Methods, 7, 41-63.

Ferrer, E., \& McArdle, J. J. (2004). An experimental analysis of dynamic hypotheses about cognitive abilities and achievement from childhood to early adulthood. Developmental Psychology, 40, 935-952.

Ferrer, E., McArdle, J. J., Shawitz, B. A., Holahan, J. N., Marchione, K., \& Shawitz, S. E. (in press). Longitudinal models of developmental dynamics between reading and cognition from childhood to adolescence. Developmental Psychology

Ferrer, E., Salthouse, T. A., Stewart, W. F., \& Schwartz, B. S. (2004). Modeling age and retest processes in longitudinal studies of cognitive abilities. Psychology and Aging, 19, 243-259.

French, J. W., Ekstrom, R. B., \& Price, L. A. (1963). Kit of reference tests for cognitive factors. Princeton, NJ: Educational Testing Service.

Fry, A., \& Hale, S. (1996). Processing speed, working memory, and fluid intelligence: Evidence for developmental cascade. Psychological Science, 7, 237-241.

Giedd, J. N., et al. (1999). Brain development during childhood and adolescence: A longitudinal MRI study. Nature Neuroscience, 2, 861-863.

Guo, S. S., Chumlea, W. C., Roche, A. F., \& Siervogel, R. M. (1997). Age- and maturity-related changes in body composition during adolescence into adulthood: The Fels Longitudinal Study. International Journal of Obesity, 21, 1167-1175.

Kail, R. (1986). Sources of age differences in speed of processing. Child Development, 57, 969-987.
Kail, R. (1988). Developmental functions for speeds of cognitive processes. Journal of Experimental Child Psychology, 45, 339-364.

Kail, R. (1991). Developmental change in speed of processing during childhood and adolescence. Psychological Bulletin, 109, 490-501.

Kail, R. (1997). Phonological skill and articulation time independently contribute to the development of memory span. Journal of Experimental Child Psychology, 67, $57-68$

Kail, R., \& Hall, L. K. (1999). Sources of developmental change in children's word-problem performance. Journal of Educational Psychology, 91, 660-668.

Kail, R., Hall, L. K., \& Caskey, B. J. (1999). Processing speed, exposure to print, and naming speed. Applied Psycholinguistics, 20, 303-314.

Kail, R. V. (2007). Longitudinal evidence that increases in processing speed and working memory enhance children's reasoning. Psychological Science, 18, 312-313.

Luna, B., Garver, K. E., Urban, T. A., Lazar, N. A., \& Sweeney, J. A. (2004). Maturation of cognitive processes from late childhood to adulthood. Child Development, 75, 1357-1372.

Mazur, J. E., \& Hastie, R. (1978). Learning as accumulation: A reexamination of the learning curve. Psychological Bulletin, 85, 1256-1274.

McArdle, J. J., Ferrer-Caja, E., Hamagami, F., \& Woodcock, R. W. (2002). Comparative longitudinal structural analyses of the growth and decline of multiple intellectual abilities over the life span. Developmental Psychology, 38, $115-142$.

McBride-Chang, C., \& Kail, R. V. (2002). Cross-cultural similarities in the predictors of reading acquisition. Child Development, 73, 1392-1407.

Miller, L. T., \& Vernon, P. A. (1997). Developmental changes in speed of information processing in young children. Developmental Psychology, 33, 549-554.

Newell, A., \& Rosenbloom, P. S. (1981). Mechanisms of skill acquisition and the law of practice. In J. R. Anderson (Ed.), Cognitive skills and their acquisition (pp. 1-51). Hillsdale, NJ: Lawrence Erlbaum Associates.

Parker, J., Bahrick, L. E., Fivush, R., \& Johnson, P. (2006). The impact of stress on mothers' memory of a natural disaster. Journal of Experimental Psychology: Applied, 12, $142-154$.

Rickard, T. C. (2004). Strategy execution in cognitive skill learning: An item-level test of candidate models. Journal of Experimental Psychology: Learning, Memory, and Cognition, 30, 65-82.

Shrager, J., \& Siegler, R. S. (1998). SCADS: A model of children's strategy choices and strategy discoveries. Psychological Science, 9, 405-410.

Wagenmakers, E. -J., \& Farrell, S. (2004). AIC model selection using Akaike weights. Psychonomic Bulletin $\mathcal{E}$ Review, 11, 192-196. 